\title{
Human Coronaviruses: The Deadly Seven
}

\section{Jonathan Daniel Hulse*}

Department of Biology and Department of Computer Science, Mathematics, and Engineering, Shepherd University, United States of America

*Corresponding Author: Jonathan Daniel Hulse, Department of Biology and Department of Computer Science, Mathematics, and Engineering, Shepherd University, United States of America.
Received: April 10, 2020

Published: May 18, 2020

(C) All rights are reserved by Danish I Jasnaik and Basilin Benson.

\section{Abstract}

Coronaviruses belong to the Family Coronaviridae, which is a classification of enveloped virus pathogens that have a large positive RNA genome. Coronaviruses are known to cause a variety of debilitating ailments including gastroenteritis and diseases of the lower/upper respiratory track. Coronaviruses are known to infect bats, cats, camels, chickens, cows, dogs, ferrets, horses, mice, minks, pigs, rabbits, raccoons, rats, snakes, tigers, and turkeys. To date, at least seven known human coronaviruses exist including: HCoV-229E, HCoV-OC43, HCoV-HKU1, HCoV-NL63, MERS-COV, SARS-CoV and the most recently discovered, SARS-COV-2, also known as COVID-19. This manuscript is intended to identify the health concerns and molecular identification methods for all known human coronaviruses in order to combat the spread of SARS-COV-2.

Keywords: Coronavirus; SARS-COV-2; COVID-19; Virus; Virology; Public Health; Molecular Biology

\section{Introduction}

Coronaviruses were first discovered in the 1960's and they belong to the Order Nidovirales, and Family Coronaviridae [59,26,34]. They are described to be enveloped virus pathogens that have the largest positive RNA genome of any viruses known to date $[5-9,26,34]$. The majority of coronaviruses are known to cause a variety of acute diseases including gastroenteritis and diseases of the upper/lower respiratory track [1-42]. Although coronaviruses are a major health concern for humans, coronaviruses are also known to infect bats, cats, camels, chickens, cows, dogs, ferrets, horses, mice, minks, pigs, rabbits, raccoons, rats, snakes, tigers, and turkeys [1-42]. All coronaviruses can have a major economic impact on the world economy due to the extensive trade agreements between nations on all the major continents.

Coronaviruses are divided into four sub-groups based upon their molecular sequence, which include: Alphacoronavirus, Betacoronavirus, Gammacoronavirus, Deltacoronavirus [5]. The term 'Human Coronaviruses' are a common term for Alphacoronaviruses and Betacoronaviruses that use humans as their main host for replication, but they can infect other organisms as alternate hosts [5,34]. As of 2020, at least seven known human coronaviruses exist, which include; HCoV-229E, HCoV-OC43, SARS-CoV, HCoV-NL63, MERS-COV, HCoV-HKU1, and the most recently discovered, SARSCOV-2, also known as COVID-19 [5,7,34]. Early genomic research revealed that the genome of human coronaviruses is incredibly large, complex and can exceed 30,000 nucleotides in length [32].
This manuscript is designed to address the major health concerns and molecular identification methods to detect the seven known human coronaviruses, including the most recently discovered SARS-COV-2, also known as COVID-19 by the World Health Organization (WHO). The seven known human coronaviruses will be presented in the order in which they were discovered.

One type of the 'Common Cold' is caused by the human coronavirus HCoV-229E which is closely related to viruses that infect insectivorous bats in Africa, Asia, Caribbean, Europe, and North America $[26,34]$. HCoV-229E was discovered in the 1960's and is known to cause mild upper respiratory infections for normally health adults but can cause severe symptoms in the elderly, HIV patients, and the young with underdeveloped immune systems [34,35]. Recent molecular dating methodologies suggest that HCov-229E evolved between the years 1346 B.C.E and 116 B.C.E. [26]. Villamil-Gómez., et al. suggests that the anti-viral drugs designed to treat HCoV-229E are unreliable and the only available treatments are to provide breathing support to the infected person [35]. Molecular identification methodologies to detect HCoV-229E have been provided by Stephensen and colleagues [31,34].

A second type of the 'Common Cold' is caused by the human coronavirus HCoV-OC43 which is closely related to HCoV-229E [4,34]. Like HCoV-229E, HCoV-OC43 was discovered in the 1960's and are closely related to viruses that infect insectivorous bats $[19,34]$. HCoV-OC43 causes acute upper respiratory infections in heathy adults, but can cause elevated symptoms in the elderly, the 
immunocompromised, and infants [20,34]. It has also been shown that HCoV-OC43 is more likely to cause lower respiratory infections in children, than other human coronaviruses [4]. Infections from $\mathrm{HCoV}-\mathrm{OC} 43$, like HCoV-229E, generally occur during the winter and early spring months $[4,20]$. Arnold and colleagues state that there is little research data related to the community spread of human coronaviruses [4]. Molecular identification methodologies to detect HCoV-OC43 have been provided by Stephensen and colleagues [31,34].

The last type of the 'Common Cold' is caused by the human coronavirus HCoV-NL63, which is a virus that is most closely related to HCoV-229E $[25,33,37,41]$. The human coronavirus HCoVNL63 can also cause 'Croup' in children and is generally thought to cause more severe clinical symptoms than HCoV-229E and HCoV-OC43 [20]. HCoV-NL63 can also cause rhinorrhea, cough, tachypnea, and obstructive laryngitis [33]. Like HCoV-229E and HCoV-OC43, HCoV-NL63 causes acute upper respiratory infections in heathy adults, but can cause elevated symptoms in the elderly, the immunocompromised, and infants [25]. The genome of HCoVNL63, like other coronavirus, is large, complex, and ranges from 28-32k base pairs [33]. Tsai and colleagues have recently shown anti-viral action of leaf extracts from Strobilanthes cusia [Nees], a plant belonging to the Acanthaceae family, which inhabits east Asia and the Indian subcontinent [33]. Although anti-viral drugs are being developed, there are currently no available cures of HCoVNL63 to date [33]. Molecular identification methodologies to detect HCoV-NL63 have been provided by various research groups $[31,33,34,41]$.

In 2002, Severe Acute Respiratory Syndrome Coronavirus, also known as SARS-CoV, became a world heath problem as it spread throughout China and other countries in east-Asia [6,11,25,34]. By 2003, the World Health Organization identified over 2353 cases of SARS-COV and reported a $4.0 \%$ mortality rate [11]. As of 2020, 8098 cases of SARS-COV was reported, and of those infected with the virus, 774 fatalities occurred, resulting in a $9.5 \%$ mortality rate [42]. Genetically, SARS-COV belongs to the genus betacoronavirus and is classified into the subgenera of Sarbecovirus [6]. Recently, Kampf and colleagues reviewed the persistence of SARS-COV on inanimate surfaces ranging from disposable gowns, to surgical gloves, to metal surfaces, showing that this virus can last on some surfaces for up to 9 days [12]. Anti-viral drugs have been developed for SARS-COV, although the success of these drugs has not resulted in a cure for this disease [42]. Identification methodologies are provided by Drosten and colleagues $[11,42]$.

HCoV-HKU1 is a human coronavirus first isolated and described from a pneumonia patient in January of 2005 [30]. Although this coronavirus does not have a common name, it has been shown to cause upper and lower respiratory tract infections in east-Asia, North America, as well as in Australia [4,25,30]. Sloots and colleagues revealed that $3.1 \%$ of upper/lower respiratory tract in- fections in Australia were caused by HCoV-HKU1 [30]. Sloots and colleagues also show that HCoV-HKU1 can be categorized into two sub-categories: type A, and type B [30]. It has also been shown by Sloots., et al. that HCoV-HKU1 is more likely to occur in the absents of other microbes and is most likely to affect children under the age of two [30]. Recently, Arnold., et al. has shown nine infections of HCoV-HKU1 in Louisville Kentucky, which accounted for less than $0.5 \%$ of the human coronavirus infections sampled from nine hospitals [4]. Molecular identification methodologies to detect $\mathrm{HCoV}$ HKU1 have been provided by Sloots research group [30].

In 2012, a novel human coronavirus first named HCoV-EMC, was re-named MERS-COV, was isolated from two infected patients from Saudi Arabia [8,25,37]. By 2013, thirteen patients had been infected with MERS-COV and seven of those patients have died showing a mortality rate of 53.84\% [27]. In 2014, 635 laboratory confirmed cases of MERS-COV existed and 193 of these cases resulted in a fatality, proving a mortality rate of $30.39 \%$ [1]. In 2015 MERS-COV spread to South Korea, causing 186 cases and 36 related deaths [21]. By 2020, 2500 cases of MERS-COV had been reported with a mortality rate of around 35\% [10]. Symptoms of MERS-COV include severe respiratory distress and, in some cases, chronic renal disfunction, diabetes mellitus, hypertension, chronic kidney disease, and chronic cardiac disease had been reported $[2,27]$. About $9.8 \%$ of infect patients of MERS-COV were recognized as asymptomatic through screening processes [3]. After molecular characterization MERS-COV, it is reported to be most related to European bat coronaviruses HCoV-HKU4 and HCoV-HKU5, isolated from samples collected in the Netherlands [27]. Investigation into carriers of MERS-COV reveal numerous non-human hosts [6]. Various anti-viral drugs have been researched for their efficacy against MERS-COV, but none of these treatments provided immunity to MERS-COV [14,29]. Identification methodologies to detect MERSCOV has been provided by numerous research groups $[8,27]$.

In December of 2019, a novel coronavirus, officially named SARS-CoV-2 by the International Committee of Taxonomy of Viruses, first appeared in Wuhan China then subsequently disseminated through-out Australia, the rest of Asia, Europe, North America, and South America [3,7,9,12-18,22-24,28,38,39,41,42]. The World Health Organization named unofficially named this novel coronavirus COVID-19, while other research groups refer to this virus as 2019-nCOV, or Wuhan $\operatorname{CoV}[3,5,9,12,24,38,39]$. The physiological effects of SARS-COV-2 very greatly, from asymptomatic patients, to patients that experiences pneumonia-like symptoms, to complete respiratory failure and death $[3,39]$. To date, no anti-viral drugs have been developed to specifically combat SARS-COV-2, so antiviral treatments designed to treat MERS-COV infections are currently being tested as a therapy for SARS-CoV-2 [14]. Identification methodologies are provided by various research groups [9,38]. 
As of March 2020, the World Health Organization has declared that SARS-COV-2, also known as COVID-19, has reached pandemic stages, and the fate of millions of people's lives is unknown. Tsai and colleagues have shown the anti-viral properties of Strobilanthes cusia [Nees], which could be studied to combat SARS-COV-2 [33]. This manuscript is intended to provided health care and public health professionals sufficient background information on all 7 known coronaviruses, in order to educate the public on the social concerns related to this virus outbreak [1-42]. The information provided in this manuscript is also intended to clarify terminology associated with human coronaviruses and provide a clear distinction between all of the known human coronaviruses [1-42]. Continued research on SARS-COV-2 will take place until the virus is contained, cured, or has run its course through the world's populations.

\section{Conclusion}

Human Coronaviruses are a group of viruses that infect humans and alternate hosts. To date, only seven coronaviruses are known to infect humans, and only a few of these are of serious consequence at the present time. This review is intended to provided background information on all seven known coronaviruses and to provided identification methods for every virus in this study. This study also mentions possible treatments for SARS-COV-2, which has become a worldwide pandemic.

\section{Acknowledgements}

No external funding was provided for this study. No internal funding was provided for this study. The single author was the only researcher who engaged in the production of this manuscript. Author declares no conflict of interests. All information provided in this manuscript shall be used by referencing this study. All of the data is available in the main text.

\section{Conflict of Interest}

No conflict of interest is declared.

\section{Bibliography}

1. Abroug Fekri., et al. "Family cluster of Middle East respiratory syndrome coronavirus infections, Tunisia, 2013". Emerging Infectious Diseases 20.9 (2014): 1527.

2. Alanazi Khalid H., et al. "Diabetes Mellitus, Hypertension, and Death among 32 Patients with MERS-CoV Infection, Saudi Arabia”. Emerging Infectious Diseases 26.1 (2020): 166.

3. Al-Tawfiq, Jaffar A. "Asymptomatic coronavirus infection: MERS-CoV and SARS-CoV-2 (COVID-19)". Travel Medicine and Infectious Disease (2020).

4. Arnold Forest W., et al. "Endemic Human Coronaviruses in Hospitalized Adults with Community-Acquired Pneumonia: Results from the Louisville Pneumonia Study". Journal of Respiratory Infections 4.1 (2020): 1.
5. Ashour Hossam M., et al. "Insights into the recent 2019 novel Coronavirus (SARS-CoV-2) in light of past human coronavirus outbreaks". Pathogens 9.3 (2020): 186.

6. Bonilla-Aldana D Katterine., et al. "MERS-CoV and SARS-CoV Infections in Animals: A Systematic Review and Meta-Analysis of Prevalence Studies". Preprints (2020).

7. Chan Jasper Fuk-Woo., et al. "Genomic characterization of the 2019 novel human-pathogenic coronavirus isolated from a patient with atypical pneumonia after visiting Wuhan". Emerging Microbes and Infections 9.1 (2020): 221-236.

8. Corman Victor., et al. "Detection of a novel human coronavirus by real-time reverse-transcription polymerase chain reaction". Eurosurveillance 17.39 (2012).

9. Corman Victor M., et al. "Detection of 2019 novel coronavirus (2019-nCoV) by real-time RT-PCR.” Eurosurveillance 25.3 (2020): 20285.

10. De Wit Emmie., et al. "Prophylactic and therapeutic remdesivir (GS-5734) treatment in the rhesus macaque model of MERSCoV infection". Proceedings of the National Academy of Sciences 117.12 (2020): 6771-6776.

11. Drosten Christian., et al. "Identification of a novel coronavirus in patients with severe acute respiratory syndrome". New England Journal of Medicine 348.20 (2003): 1967-1976.

12. Kampf, G., et al. "Persistence of coronaviruses on inanimate surfaces and its inactivation with biocidal agents". Journal of Hospital Infection (2020).

13. Khot Wasim Yunus and Milind Y Nadkar. "The 2019 Novel Coronavirus Outbreak-A Global Threat". The Journal of the Association of Physicians of India 68.3 (2020): 67-71.

14. Ko Meehyun., et al. "Screening of FDA-approved drugs using a MERS-CoV clinical isolate from South Korea identifies potential therapeutic options for COVID-19". Bio Rxiv (2020).

15. Kissler S., et al. Projecting the transmission dynamics of SARSCoV-2 through the post-pandemic period (2020).

16. Li Xiang., et al. "Bat origin of a new human coronavirus: there and back again". Science China Life Sciences 63.3 (2020): 461462.

17. Lorusso Alessio., et al. "Novel coronavirus (SARS-CoV-2) epidemic: a veterinary perspective". Veterinaria Italiana (2020).

18. Meo SA., et al. "Novel coronavirus 2019-nCoV: prevalence, biological and clinical characteristics comparison with SARS-CoV and MERS-CoV". European Review for Medical and Pharmacological Sciences 24 (2020): 2012-2019.

19. Nilsson Anna., et al. "Fatal encephalitis associated with coronavirus OC43 in an immunocompromised child". Infectious Diseases (2020): 1-4 
20. Niu Junwei., et al. "Non-invasive bioluminescence imaging of HCoV-OC43 infection and therapy in the central nervous system of live mice". Antiviral Research 173 (2020): 104646.

21. Noh Jin-Won., et al. "Effect of Information Disclosure Policy on Control of Infectious Disease: MERS-CoV Outbreak in South Korea". International Journal of Environmental Research and Public Health 17.1 (2020): 305.

22. Pan Yang., et al. "Viral load of SARS-CoV-2 in clinical samples". The Lancet Infectious Diseases 20.4 (2020): 411-412.

23. Paraskevis Dimitrios., et al. "Full-genome evolutionary analysis of the novel corona virus (2019-nCoV) rejects the hypothesis of emergence as a result of a recent recombination event". Infection, Genetics and Evolution 79 (2020): 104212.

24. Phan Tung. "Novel coronavirus: From discovery to clinical diagnostics". Infection, Genetics and Evolution 79 (2020): 104211

25. Pérez Cecilia Perret and Marcela Ferrés Garrido. "Pneumonia Caused by Emerging Viral Agents". Pediatric Respiratory Diseases (2020): 335-341.

26. Pfefferle Susanne., et al. "Distant relatives of severe acute respiratory syndrome coronavirus and close relatives of human coronavirus 229E in bats, Ghana". Emerging Infectious Diseases 15.9 (2009): 1377.

27. Raj V Stalin., et al. "Dipeptidyl peptidase 4 is a functional receptor for the emerging human coronavirus-EMC". Nature 495.7440 (2013): 251-254

28. Rasheed Muhammad Asif., et al. In Silico Identification of Novel B Cell and T Cell Epitopes of Wuhan Coronavirus (2019. nCoV) for Effective Multi Epitope-Based Peptide Vaccine Production (2020).

29. Sheahan Timothy P., et al. "Comparative therapeutic efficacy of remdesivir and combination lopinavir, ritonavir, and interferon beta against MERS-CoV". Nature Communications 11.1 (2020): 1-14.

30. Sloots Theo P., et al. "Evidence of human coronavirus HKU1 and human bocavirus in Australian children". Journal of Clinical Virology 35.1 (2006): 99-102.

31. Stephensen Charles B., et al. "Phylogenetic analysis of a highly conserved region of the polymerase gene from 11 coronaviruses and development of a consensus polymerase chain reaction assay". Virus Research 60.2 (1999): 181-189.

32. Thiel Volker., et al. "Infectious RNA transcribed in vitro from a cDNA copy of the human coronavirus genome cloned in vaccinia virus". Journal of General Virology 82.6 (2001): 12731281.
33. Tsai Yu-Chi., et al. "Antiviral Action of Tryptanthrin Isolated from Strobilanthes cusia Leaf against Human Coronavirus NL63". Biomolecules 10.3 (2020): 366.

34. Van der Hoek Lia., et al. "Identification of a new human coronavirus". Nature Medicine 10.4 (2004): 368-373.

35. Villamil-Gómez, Wilmer E., et al. "Fatal human coronavirus 229E (HCoV-229E) and RSV-Related pneumonia in an AIDS patient from Colombia". Travel medicine and infectious disease (2020): 101573

36. Wang, Lin-Fa., et al. "From Hendra to Wuhan: what has been learned in responding to emerging zoonotic viruses". The Lancet 395.10224 (2020): e33-e34

37. Wang, Yanqun., et al. "Discovery of a subgenotype of human coronavirus NL63 associated with severe lower respiratory tract infection in China, 2018". Emerging Microbes and Infections 9.1 (2020): 246-255.

38. World Health Organization. "Laboratory testing for coronavirus disease 2019 (COVID-19) in suspected human cases: interim guidance 2020..' World Health Organization (2020).

39. Xu Xintian., et al. "Evolution of the novel coronavirus from the ongoing Wuhan outbreak and modeling of its spike protein for risk of human transmission". Science China Life Sciences 63.3 (2020): 457-460.

40. Zhang Jiahao., et al. "The continuous evolution and dissemination of 2019 novel human coronavirus". Journal of Infection (2020): 30083-30089

41. Zhang Lu., et al. "Complete Genome Sequences of Five Human Coronavirus NL63 Strains Causing Respiratory Illness in Hospitalized Children in China". Microbiology Resource Announcements 9.8 (2020)

42. Zhou Yadi., et al. "Network-based Drug Repurposing for Human Coronavirus". Med Rxiv (2020).

\section{Assets from publication with us}

- Prompt Acknowledgement after receiving the article

- Thorough Double blinded peer review

- Rapid Publication

- Issue of Publication Certificate

- High visibility of your Published work

Website: https://www.actascientific.com/

Submit Article: https://www.actascientific.com/submission.php Email us: editor@actascientific.com

Contact us: +919182824667 